\title{
« Pour obtenir de l'aide, appuyez sur X ». La montée du paradigme d'assistance dans le design du jeu vidéo
}

\section{Carl Therrien et Mikaël Julien}

\section{OpenEdition}

\section{Journals}

Édition électronique

URL : http://journals.openedition.org/sdj/508

DOI : $10.4000 /$ sdj. 508

ISSN : 2269-2657

Éditeur

Laboratoire EXPERICE - Centre de Recherche Interuniversitaire Expérience Ressources Culturelles Education

\section{Référence électronique}

Carl Therrien et Mikaël Julien, « «Pour obtenir de l'aide, appuyez sur X». La montée du paradigme d'assistance dans le design du jeu vidéo », Sciences du jeu [En ligne], 4 | 2015, mis en ligne le 05 octobre 2015, consulté le 06 avril 2021. URL : http://journals.openedition.org/sdj/508 ; DOI : https:// doi.org/10.4000/sdj.508

Ce document a été généré automatiquement le 6 avril 2021.

\section{c) 98}

La revue Sciences du jeu est mise à disposition selon les termes de la Licence Creative Commons Attribution - Pas d'Utilisation Commerciale - Pas de Modification 4.0 International. 


\title{
« Pour obtenir de l'aide, appuyez sur X ». La montée du paradigme d'assistance dans le design du jeu vidéo
}

\author{
Carl Therrien et Mikaël Julien
}

\section{NOTE DE L'AUTEUR}

Une première version de cet article est parue en anglais dans The Handbook of Digital Games (Therrien, 2014). Cette traduction française a été modifiée et bonifiée par les deux auteurs. Notamment, une nouvelle mise en contexte académique a remplacé l'introduction originale, et la section « Baliser la progression » est construite à partir d'observations inédites.

1 L'échec constitue l'élément central de l'art ludique; c'est ce qu'affirme de manière péremptoire Jesper Juul dans son ouvrage récent The Art of Failure (2013). En toute bonne foi, il fait l'effort de comparer ce nouvel art avec les formes plus traditionnelles, afin d'en cerner l'originalité : «Les jeux sont significatifs non seulement parce qu'ils représentent des tragédies, mais aussi parce qu'ils engendrent occasionnellement de véritables tragédies personnelles » (Juul, 2013, p. 26). ${ }^{1}$ Alors qu'il reconnait d'emblée une certaine disparité chez les joueurs et la nature polymorphe des œuvres vidéoludiques, son argumentaire demeure somme toute normatif : « les jeux vidéo sont l'art de l'échec, la seule forme artistique qui nous prépare à l'échec et nous permet de vivre et d'expérimenter avec l'échec » (Juul, 2013, p. 30).

2 Comme le précise Juul (2013, p. 29), la tolérance envers l'échec n'est pas une donnée stable dans l'histoire du jeu vidéo. Or, certaines observations récentes tendent à démontrer que cette tolérance atteint parfois un point d'achoppement qui semble remettre en cause la prémisse fondamentale de son ouvrage. Dans sa critique récente 
de Another World (Delphine Software, 1991; édition $20^{\mathrm{e}}$ anniversaire parue en 2011/2014), Colin Moriarty (2014) indique en conclusion : " Another World est considéré comme un classique d'un âge d'or du jeu. Je ne comprends pas pourquoi ». Plus spécifiquement, c'est la difficulté et le «mauvais design" qui motivent ce rejet expéditif. On retrouve une mépréhension similaire dans l'article que Alasdair Duncan consacre au dernier jeu de la série des Tex Murpthy, Tesla Effect (Big Finish Games, 2014). L'auteur note quelques "concessions au design moderne " notamment avec l'inclusion d'un système d'indices (Duncan, 2014), alors que Under a Killing Moon (Access Software, 1994) contenait déjà un tel système. Le journaliste associe le jeu d'aventure de l'époque à une difficulté sans concession, typique de l'avant "révolution casual » (Juul, 2009). À la lumière de ces observations, il apparaît évident qu'une césure existe entre la réception de ces jeux à l'époque de leur parution, où ils ont été encensés et rapidement considérés comme des œuvres phares, et celle d'un public contemporain qui, n'ayant pas connu ces jeux en leur temps, leur jette un regard acerbe.

3 Alors que Rules of Play (2003) insiste également sur l'importance du conflit et de la compétition, Katie Salen et Eric Zimmerman mettent en avant un autre concept qui vient tempérer un peu cet élément de définition : « la généralisation de la compétition dans les jeux ne signifie pas que ces derniers ne sont pas aussi coopératifs. Même si nous pouvons affirmer avec confiance que tous les jeux sont compétitifs, il est tout autant vrai que tous les jeux sont coopératifs » (2003, p. 275). Ils poursuivent : "L'idée que les jeux sont à la fois compétitifs et coopératifs est contradictoire seulement si les deux termes s'excluent mutuellement, ce qui n'est pas le cas. La racine latine du mot "compétition" est con petire, qui veut dire "chercher ensemble" » (2003, p. 276). Les auteurs comparent l'expérience du jeu à l'apprentissage commun d'un langage, dans le but de créer un «discours » cohérent et de faciliter la gratification et le caractère significatif de l'activité.

4 Les changements de culture - aussi bien théoriques que ludiques - que nous venons de relever sont imputables à une variété de facteurs. Dans cet article, nous n'adopterons pas une attitude " ontologisante » qui chercherait à attribuer un statut plus essentiel à l'une ou l'autre des composantes de l'activité ludique. Nous nous contenterons plus simplement de documenter ce qui apparaît comme une évidence historique : depuis l'arrivée du jeu vidéo sur nos écrans, le « discours » de la communauté ludique évolue, au point où certains acteurs ne semblent plus "parler» le même langage. Afin de mieux cerner ces évolutions, nous tenterons de comprendre l'influence de l'expérience jouable - et plus particulièrement la systématisation de l'assistance prodiguée en cours de route - sur les attentes des joueurs. Nous croyons que ces changements permettent d'expliquer en grande partie l'incompréhension qui semble accabler les évaluateurs contemporains de jeux classiques réputés pour leur difficulté.

5 À partir du modèle proposé par les auteurs de Digital Play, nous ferons état de l'évolution de l'expérience vidéoludique en explicitant un grand nombre d'interactions entre les circuits industriel, technologique et culturel (Kline et al., 2003). Une première section («Émergence de la progression») s'attachera à décrire ces interactions en relation avec les deux impératifs de design dont il a été question en ouverture: antagonisation et coopération. Comme le notent Salen et Zimmermann, ces deux impératifs sont présents dans tout jeu, mais nous défendrons l'hypothèse d'un développement historique qui tend à favoriser une interpellation coopérative, relayée par de multiples interactions entre les circuits industriel, technologique et culturel. 
Alors que l'« âge d'or" du jeu repose en grande partie sur la gratification sociale engendrée par la maîtrise de défis ludiques (et répercutée par un score affiché publiquement) dans un espace d'exploitation public (l'arcade), la conquête progressive de l'espace domestique permettra aux concepteurs de proposer des univers plus riches et une expérience unique qui s'étend sur de nombreuses heures de jeu. Au-delà du plaisir de surmonter les défis et de l'éventuel capital social associé à cette maîtrise, le jeu de progression (Juul, 2005) propose d'autres gratifications intrinsèques, et éventuellement une gratification "ultime »: compléter un récit. Nous présenterons également l'émergence du fantasme de puissance dans le discours publicitaire, qui acquiert une grande visibilité et vient tempérer une antagonisation ludique toujours très vive au cours des années 1980 .

6 Dans une deuxième section ("De l'aide en série »), nous décrirons l'apparition et l'évolution des différents systèmes d'assistance, qui rend manifeste l'équilibre précaire et constamment renégocié entre antagonisation et coopération. Pour bien comprendre l'intérêt de ces systèmes et expliquer leur portée cognitive, nous nous référerons aux principes de l'apprentissage idéalisé tels que définis par James Paul Gee. Celui-ci, dans son ouvrage What Video Games Have to Teach Us About Learning and Literacy (Gee, 2003), souligne la grande expertise développée par les artisans de l'industrie du jeu à travers les décennies pour faciliter l'apprentissage au cours d'une expérience interactive. Afin de bien relever l'évolution, nous avons décidé de présenter plusieurs systèmes à travers quelques séries de jeux populaires. Dans un premier temps, nous présenterons les différents moyens qui visent à faciliter l'apprentissage du joueur (indices, didacticiels), notamment à travers plusieurs jeux de la franchise Batman. Dans un second temps, nous nous nous intéresserons aux marqueurs de progression (émergence du checkpoint, carte de progression et items à collectionner dans la série Donkey Kong Country), et à l'émergence de différents systèmes de guidage spatio-narratif (cartes à compléter, radar dynamique, indicateurs directionnels).

\section{Émergence de la progression}

7 Pour mettre en contexte notre étude de la dynamique antagonisation / coopération dans le design de jeu vidéo, nous devons prendre acte de facteurs socioéconomiques et matériels qui ont influencé cette pratique de design à ses débuts. Comme l'indique Van Burnham (2003, p. 56), le concept de jeux électroniques jouables sur téléviseur dans le confort domestique était complètement nouveau - et par conséquent difficile à commercialiser - au tournant des années 1970. Toutefois, le fait de s'adonner à des activités ludiques dans une arcade ou un bar faisait déjà partie des mœurs étatsuniennes depuis des lustres. Au cours des années 1930 et 1940 à Chicago, un modèle industriel lucratif et stable émerge, avec des corporations phares (Bally, Midway, Williams, Gottlieb, Chicago Coin), des lieux d'exploitation reconnus et des intermédiaires s'occupant de l'entretien des machines. Après des décennies de divertissement public décliné sous de nombreuses formes (jukebox, théâtres mécaniques, novelty games et surtout les flippers popularisés au cours des années 1940), cette industrie allait être dynamisée de nouveau par l'arrivée du jeu vidéo. L'arcade constituait l'espace de commercialisation le plus logique pour mettre en marché les nouvelles machines. L'opération individuelle et automatisée grâce à la monnaie - le fameux coin-op - était idéale pour rentabiliser les nouvelles technologies attractives : 
une machine populaire pouvait facilement rapporter des centaines de pièces de 25 cents par jour. Après la tentative infructueuse de Bill Pitts et Hugh Tuck de transposer Spacewar! sous la forme d'un double cabinet, le lancement des machines Computer Space (1971) et PONG (1972) ouvriront la porte à une expansion commerciale fulgurante. En tant que lieu de socialisation, d'attraction technologique et d'innovation ludique, l'arcade a profondément influencé la culture ludique. Comme nous le verrons dans cette section, ce lieu a grandement favorisé une expérience de jeu basée sur la compétition.

8 Sur la boîte de l'adaptation sur console de salon de Jungle Hunt (Taito, 1983), on peut lire que " cette compétition correspond à la formule classique des jeux à succès : facile à apprendre, difficile à maîtriser ». Ce dicton est souvent utilisé pour résumer la conception du design associée aux jeux d'arcade classiques. En effet, on peut dire de ces jeux qu'ils sont d'une simplicité trompeuse: la facilité d'utilisation au début de l'expérience est largement contrée par la difficulté de la progression.

\section{La course en avant}

9 Dans la logique de l'arcade, un jeu n'a pas avantage à définir clairement des conditions de réussites qui viendraient clore définitivement l'expérience. Beaucoup de vieux classiques n'ont d'ailleurs pas de conclusion officielle, optant plutôt pour une difficulté qui augmente continuellement par le truchement de modifications algorithmiques d'un niveau à l'autre. Les contraintes technologiques de l'époque favorisaient aussi cette approche; une même scène ludique pouvait occuper un joueur plus longtemps sans avoir à stocker de nouveaux éléments. Les kill screens de jeux comme Pac-Man (Namco, 1980) ou Donkey Kong (Nintendo, 1981), c'est-à-dire des niveaux infranchissables et souvent corrompus visuellement, correspondent à un échec de cette création algorithmique, et rendront perceptibles les failles de ce design face à la compétence grandissante de certains joueurs experts. L'expérience ludique optimale des premiers temps a été conçue pour se solder systématiquement par la "mort ». Même lorsque des conclusions intentionnelles ont commencé à être implémentées dans des jeux comme Crystal Castles (Atari, 1983), la complétude de l'expérience représentait en soi un exploit inhabituel. Après le dixième niveau, le joueur est récompensé par la capitulation du logiciel: "J'abandonne: tu gagnes. Tu dois être un génie du jeu vidéo", le tout accompagné d'une version électronique de l'Ouverture 1812 de Tchaïkovski, musique triomphante par excellence.

Au-delà des défis ponctuels, l'objectif de ces jeux était de démontrer son talent par rapport à celui des autres joueurs. En ce sens, la première position du palmarès des high scores représentait le véritable objectif englobant. Le concept d'un pointage comparatif est de toute évidence un héritage des pratiques sportives, et était présent dans les arcades bien avant l'avènement des jeux vidéo. Les machines de pinball et autres dispositifs électro-mécaniques affichaient les meilleurs pointages et offraient déjà aux joueurs l'opportunité d'entrer leurs initiales. Le score - et la pratique compétitive que celui-ci encourage - faisait donc office d'agent extrinsèque de motivation, par opposition au plaisir intrinsèque $\mathrm{du}_{\mathrm{j}}$ jeu. $^{2}$ Les jeux vidéo adaptant des pratiques sportives - tels que PONG et ses nombreuses déclinaisons - intégraient cet aspect de façon naturelle, mais même des jeux basés sur des scénarios fantaisistes plus élaborés tels que Computer Space - évaluaient la performance des joueurs sous la forme d'une 
valeur numérique claire ; les actions simulées étaient traduites sous la forme d'un score afin de faciliter une pratique compétitive. En 1976, la machine de Sea Wolf (Midway) affichait les records à l'écran à la fin des parties ainsi qu'en mode "attraction », afin de mieux interpeller les joueurs à l'esprit compétitif. De nombreuses machines populaires - dont Asteroids (Atari, 1979), Tempest (Atari, 1980), Pac-Man et Donkey Kong - ont intégré des tableaux indiquant quels ennemis ou actions étaient les plus profitables à l'ego du joueur. En 1982, Walter Day lançait le Twin Galaxies National Scoreboard après avoir récolté les scores de douzaines de machines d'arcade à travers l'Amérique ; l'opération s'étendra rapidement au niveau international. Les meilleurs scores seront publiés dans le Guinness Book of World Records. Twin Galaxies organisera aussi des compétitions nationales.

De nombreux classiques des jeux d'arcade ont été convertis pour les systèmes domestiques comme l'Atari VCS. Dans les feuillets ou manuels qui accompagnent ces adaptations, les joueurs pouvaient trouver de l'information précise sur le calcul du pointage en fonction de certaines actions. Ces manuels incluaient habituellement des pages vierges à la fin pour que les joueurs puissent y noter leurs meilleurs scores; puisqu'il était impossible d'enregistrer des informations volatiles sur les consoles de l'époque, les scores disparaissaient lorsque celles-ci étaient éteintes. L'esprit de compétition - que ce soit contre soi-même ou d'autres - était encouragé à l'extérieur de l'arcade : plusieurs jeux créés spécifiquement pour le marché domestique incorporaient également l'atteinte $\mathrm{du}$ meilleur score possible comme objectif principal. L'omniprésence de ce mode d'interpellation compétitif devient particulièrement évidente dans les jeux d'aventure populaires de Sierra On-Line. Alors que ces expériences ludiques proposaient de nouveaux plaisirs intrinsèques, King's Quest (1984), Space Quest (1986) et leurs successeurs affichaient aussi un score au haut de l'écran, incluant le score maximal à atteindre, invitant ainsi les joueurs à améliorer leur performance.

12 Au tournant des années 1980, l'émergence d'une ruse provoque un chamboulement historique bien documenté dans le design des jeux d'arcade (Kent, 2001). Peu après la sortie d'Asteroids (Atari, 1979), certains joueurs ont trouvé un moyen de maximiser leur score en exploitant une faille du système de jeu: en ne gardant qu'un seul petit astéroïde à l'écran - empêchant ainsi le jeu de progresser aux niveaux suivants, plus difficiles - le joueur pouvait continuer d'abattre les soucoupes volantes qui continuent d'apparaître périodiquement. Il pouvait ainsi accumuler des points supplémentaires ad vitam aeternam, et monopoliser une machine pendant de longues minutes. Ce bouleversement du modèle commercial par les joueurs experts entraînera l'émergence d'une caractéristique qui reste, à ce jour, l'un des signes les plus évidents du paradigme de design compétitif mis en avant à l'arcade, et que nous avons choisi de nommer «index punitif »: un élément perceptible vient signifier l'impératif de la course en avant perpétuelle, à défaut de quoi le système pourrait déclencher la fin de la partie en cours. Des minuteries extradiégétiques feront leur apparition dans plusieurs jeux, indiquant clairement l'inéluctable fin du plaisir technologique - à moins, bien sûr, d'insérer une nouvelle pièce de monnaie. L'appellation trouve son plein sens dans certains jeux qui l'intégreront de façon littérale. Dans The Simpsons (Konami, 1990), une main géante fait son apparition sans la moindre justification diégétique, clignotant de plus en plus rapidement au son d'une cloche, poussant littéralement les joueurs vers l'avant si ceux-ci demeurent inactifs. Certains jeux développent cette pression punitive de manière encore plus expéditive; dans Teenage Mutant Ninja Turtle s: Turtles in Time 
(1991) - une autre adaptation classique de Konami - les joueurs étaient tués par une bombe s'ils n'obéissaient pas à l'écriteau «hurry!». Dans ces exemples, le designer signifie cavalièrement son emprise sur l'expérience de jeu. Mais surtout, les impératifs du circuit industriel remontent jusqu'à la surface de l'écran, et s'installent au cœur de l'expérience culturelle.

\section{Un défi impossible}

Malgré une apparence de "plaisir pour toute la famille» communiquée par les premières campagnes publicitaires de Magnavox et d'Atari, les développeurs s'adressaient spécifiquement aux joueurs experts dans des publicités ou sur les emballages des jeux en soulignant l'ampleur du défi proposé, adoptant même la bravade pour interpeller le joueur potentiel. La borne de Space Invaders est d'ailleurs très directe à cet égard: "la partie se termine lorsque les bases des joueurs sont complètement détruites par les missiles des envahisseurs ou lorsque les envahisseurs dominent le jeu ». Dans la publicité télévisée de la version pour console de salon de Berzerk (Stern, 1982), une charmante vieille dame relève le défi posé par son petit-fils alors que le jingle d'Atari se fait entendre ; la publicité se termine par un renversement des rôles alors que la dame demande : «peux-tu faire mieux? ». Dynamique similaire pour une publicité de Ms. Pac-Man: le présentateur souligne le défi apporté par le jeu, avec son dernier labyrinthe qui est «si difficile à atteindre, vous ne le reverrez peutêtre plus jamais!». "Terminer votre patrouille en un seul morceau est quasi impossible ", assure le manuel pour la version Atari 400/800 de Moon Patrol (Irem, 1982). Dans sa première aventure vidéoludique éditée par Parker Brothers sur l'Atari VCS, un Spiderman médusé demande au spectateur : «Serait-ce là trop d'action même pour Spiderman?». Dans le dépliant du populaire jeu multiplateforme River Raid (1982), Carol Shaw nargue manifestement le joueur: «si vous parvenez à revenir en territoire allié, écrivez-moi ». D'une version à l'autre de Impossible Mission (1984), Epyx soulignait sans hésitation son " défi sans fin "; "sauver le monde n'était pas censé se faire facilement». Sur le boîtier de la réédition de Cauldron (Palace Software, 1985-1986), une série déjà bien connue pour sa difficulté monumentale, l'acheteur potentiel est informé de l'éventuel défi insurmontable, «difficile même pour les joueurs expérimentés ».

Dans un contexte d'évolution technologique rapide, la nouveauté des univers audiovisuels constituait parfois une motivation suffisante pour tenter de surmonter ces défis. «Avez-vous le courage de charger l'expérience qui donne à tous les autres jeux auxquels vous avez joué une apparence préhistorique? » (arrière du boîtier de Blood Money, DMA Design, 1989); la promesse du sublime technologique agit comme principale motivation pour faire face au «plus grand défi auquel un joueur se soit jamais confronté ». Au-delà des nombreux développements historiques présentés dans ce texte, l'attraction technologique constitue l'une des rares données constantes dans la mise en marché du jeu vidéo. Cette orientation du désir vers la puissance de la technologie ne surprend guère dans un milieu industriel marqué par l'innovation destructrice (Kline et alii, 2003) et l'obsolescence accélérée (Newman, 2012).

Comme nous l'avons vu dans cette section, l'antagonisation du joueur par la publicité était largement répandue dans une période dominée par les jeux d'arcade, et l'adaptation de ces derniers sur consoles de salon. Ce type d'interpellation demeure 
associé aux genres qui établissent un défi ludique à partir de la coordination rapide entre les yeux et les mains, mais a aussi été convoquée pour d'autres genres. Les cassetêtes alambiqués proposés par les jeux d'aventure graphiques et textuels représentaient également un bon défi au tournant des années 1980, et une compagnie comme Sierra On-Line s'assurait de bien en informer les joueurs sur les boîtes de leurs premiers classiques: "Chaque aventure Sierra procure fascination et intrigue, et nécessite plusieurs semaines pour être résolue. Alors rassemblez vos esprits et votre persévérance, car voilà une aventure de Sierra - un défi pour votre ingéniosité ». Comme le note Jonathan Lessard (2014), «dans le contexte émergent du jeu sur ordinateur personnel, les développeurs et éditeurs doivent trouver des arguments pour distinguer favorablement les jeux d'aventure", et cette distinction se fera par l'entremise du type de défi mis en avant.

\section{Le plaisir de la fin}

Les causes du krach de 1983 sont nombreuses et bien documentées (Kent, 2001 ; Wolf, 2008; Donovan, 2010). Parmi celles-ci, la frustration d'un large public face à l'augmentation de la difficulté, qui cherchait à contrebalancer les habiletés grandissantes des joueurs passionnés. Noah Falstein - l'un des designers de Sinistar (Williams, 1982), reconnu pour son intransigeance - remarque: "À mesure que les joueurs s'amélioraient, les jeux d'arcade sont devenus plus difficiles afin de maintenir un roulement de monnaie élevé » (cité dans Donovan, 2010, p. 97-98). Encore ici, l'influence des impératifs commerciaux sur le circuit culturel est évidente: afin de maximiser le «taux de roulement » devant la machine, l'inclinaison de la courbe de difficulté fut rehaussée dans plusieurs jeux. Le modèle commercial qui avait donné naissance à une industrie multimilliardaire s'est essoufflé, amenant de nombreuses arcades à fermer leurs portes et des bars et restaurants à se départir de leurs bornes d'arcade.

17 L'exploitation du jeu vidéo dans un milieu domestique bouscule l'essence même de la production et de la vente d'appareils technologiques. Le prix de fabrication et de vente ne peut être aussi élevé que pour une machine d'arcade, surtout pour un phénomène relativement nouveau. Par contre, ce type d'exploitation permet de générer du profit sans se soucier du roulement rapide de la monnaie, une fois la vente initiale effectuée. Comme Erkki Huhtamo l'a indiqué dans Before the Crash, le contexte domestique a été associé à un refuge sécuritaire depuis l'ascension de la maison bourgeoise victorienne ; les membres de la famille y sont protégés du chaos de la vie industrielle en expansion rapide (Huhtamo, 2012, p. 35). Il serait exagéré de prétendre que cette connotation culturelle a eu un impact direct sur le développement d'expériences ludiques moins punitives dans les jeux vidéo domestiques. Nous affirmerons cependant au cours de cette section que certains changements significatifs ont été apportés à l'expérience ludique en partie à cause de ce changement de milieu. Dans plusieurs genres, le rythme du jeu change. En parallèle, les stratèges marketing commencent à cibler un groupe démographique de façon plus spécifique: les enfants. Comme l'ont démontré les auteurs de Digital Play (Kline et al., 2003), la renaissance de l'industrie repose sur la création de la "génération Nintendo ».

18 À l'arrivée de Nintendo sur le marché des consoles de salon, l'évaluation des performances via le système de pointage était encore largement implémentée. 
Plusieurs jeux d'arcade populaires comme Galaga (Namco, 1985), Alien Syndrome (Sega, 1987), After Burner (Sega, 1988), Ikari Warriors (SNK, 1986), Operation Wolf (Taito, 1989) ou Target Renegade (Ocean, 1990) ont été adaptés sur la Nintendo Entertainment System (NES) ; tous mettent l'accent sur l'atteinte du meilleur score. Le manuel du célèbre Cabal (Tad Corporation, 1989), transposé sur NES en 1990, énumère deux objectifs : «1. parcourez les cinq niveaux » et «2. accumulez le plus de points possible ». Ici, nous pouvons déjà remarquer un certain glissement dans la façon d'interpeller les joueurs : les designers ont choisi de mettre l'accent sur le premier objectif, reléguant le score au second rang. Dans Rollergames (Konami, 1990) et Shatterhand (Natsume, 1991), le score est affiché à la fin de chaque niveau, et la présentation des ennemis dans le manuel n'inclut pas les traditionnelles équivalences chiffrées. Dans Batman: The Video Game, le score n'est affiché que lorsque le jeu est mis en mode "pause ». Le manuel contient une page pour prendre en note les scores, mais il apparaît raisonnable de supposer que celle-ci n'intéressait pas un grand nombre de joueurs. La notation chiffrée demeurera cardinale au déploiement d'un autre genre qui gagne en popularité à cette époque : le RPG. Cette dernière réfère alors aux points d'expérience accumulés par le joueur tout au long de sa quête; la modélisation statistique est intégrée de manière endogène à l'univers et à l'expérience du jeu.

De toute évidence, l'objectif énoncé par certains jeux n'était plus seulement de faire preuve de compétence par rapport à une performance antérieure ou aux autres joueurs, mais aussi d'atteindre un état de jeu désiré, soit de surmonter tous les obstacles du monde ludique. Dans le manuel de Ninja Gaiden (Tecmo, 1988), une section nommée "Epilogue " mentionne: "Action cinéma "Ninja Gaiden" dépend de vos habilités pour sauver le monde. Pouvez-vous le faire? Votre réussite est essentielle! Une finale que vous devez expérimenter par vous-mêmes vous attend». Bien sûr, l'atteinte de la fin de ce jeu réputé pour sa difficulté représentait en soi un exploit, conférant automatiquement au joueur un gain en capital social. Mais le plaisir de la clôture narrative est ici présenté comme une grande motivation intrinsèque; la maitrise de l'adversité - compris à la fois dans son sens diégétique, mais également dans le sens d'avoir «battu le jeu » - génère un état de plénitude affective indéniable. Opère ici une transition vers ce que Jesper Juul qualifie de « jeux de progression » dans son livre Half-Real (par opposition aux jeux d'émergence, où une infinité d'actualisations peuvent émerger à partir de quelques règles simples (Juul, 2005). Il s'agit d'un développement important d'un point de vue historique puisque, selon l'auteur, le plaisir d'évoluer dans un parcours d'obstacles préétabli n'a jamais été aussi répandu dans l'histoire du jeu avant l'arrivée des jeux vidéo contemporains. Il apparaît évident que le plaisir de la progression dans un univers de jeu construit minutieusement est devenu un aspect inhérent du plaisir offert par les jeux vidéo au cours des années 1980. Plutôt que de punir les joueurs dont la performance n'était pas satisfaisante, la « main » du designer a proposé graduellement des mesures facilitantes pour aider ces derniers à atteindre un état de jeu désiré. La transition du contexte d'exploitation privilégié (des arcades au marché domestique) ainsi que l'augmentation des budgets, du personnel créatif et des nouvelles possibilités technologiques (notamment, des capacités de stockage accrues) ont toutes contribué à cette transformation du circuit culturel. 


\section{Un discours inclusif : le héros confiant}

20 domestiques représentait un investissement risqué. Les créateurs de Donkey Kong ont vu ce désistement comme une opportunité de conquérir le marché états-unien. Nintendo avait déjà du succès au Japon avec la Famicom; le lancement de son équivalent américain allait reposer sur l'habileté de la compagnie à convaincre les revendeurs échaudés par le krach (Kent, 2001, chapitre 20 ; Donovan, 2010, chapitres 13 and 26), et sur une stratégie de marketing visant les enfants plus spécifiquement. La génération Nintendo était sur le point d'émerger (Kline et alii, 2003, chapitre 5).

Tout comme leurs prédécesseurs, les premiers grands titres à succès pour la Nintendo Entertainment System relevaient du défi insurmontable, et étaient présentés comme tel par la mise en marché. Une célèbre publicité datant des débuts de la NES présente les créatures de plusieurs jeux animés en images de synthèse; «Nous sommes Nintendo. Vous ne pouvez pas nous battre », clament d'effrayantes voix robotiques. Après avoir décrit brièvement l'univers fictionnel des jeux, les boîtiers de Super Mario Bros. (1985) et Metroid (1986) annoncent aux joueurs potentiels : « ce ne sera pas facile ». Nintendo a même présenté The Legend of Zelda (1986) comme étant « le jeu vidéo le plus difficile que nous ayons jamais créé». L'orgueil des joueurs était constamment interpelé par le truchement d'une simple interrogation : «Avez-vous ce qu'il faut pour sauver la Princesse Champignon?» (Super Mario Bros.) ; "Est-ce que vous parviendrez avec Mega Man à pénétrer les sept sociétés différentes du Dr. Wily et à préserver la race humaine?» (Mega Man, Capcom, 1987). Certains classiques plébiscités à l'époque faisaient déjà montre d'une attitude plus confiante envers les habiletés du joueur. Le manuel du second opus de la série Mega Man (Capcom, 1988) précise notamment : «La civilisation repose sur vos habiletés et votre courage - assure que vous, MegaMan, serez encore debout à la fin ». Une évolution cardinale de la culture ludique allait justifier un changement généralisé de rhétorique : la puissance enfin conférée aux joueurs.

Durant la première expansion des jeux vidéo à l'arcade, il était commun de voir imprimé sur le cabinet une liste décrivant les différentes actions possibles et les armes disponibles dans le jeu. En 1980, une mécanique de jeu innovatrice gagnera rapidement en popularité : le power-up. Dans Pac-Man, les power pellets renversent la dynamique poursuivant/poursuivi, permettant à cette première star vidéoludique de chasser Blinky, Pinky, Inky et Clyde, amassant des points par la même occasion. Cette fonctionnalité allait rapidement être mise à l'avant-plan du marketing de jeux vidéo. On retrouve déjà ce fantasme de puissance dans la présentation des premiers jeux de rôle informatisés, fondés sur l'acquisition d'armement, de sorts et d'objets magiques. Le boîtier d'Ultima II relève cet aspect comme une attraction majeure : «développez et maniez la magie la plus puissante connue de l'homme ». Cette mise en puissance discursive sera disséminée durant l'ascension de l'empire Nintendo, à commencer par le nouveau slogan proposé par la compagnie en 1986 : «Maintenant vous jouez avec le pouvoir ». " Mario est de retour!", déclare le boîtier de Super Mario Bros. 2 (version occidentale, Nintendo, 1988), "plus grand et plus méchant que jamais! ", alors que l'arrière de la boîte de Super Mario Bros. 3 (Nintendo, 1990) souligne les habits animaliers qui confèrent de nouvelles habiletés au populaire héros. De nouveaux super héros maniant d'incroyables pouvoirs seront popularisés sur cette plateforme. Outre les figures de proue que sont Mario et Mega Man, les joueurs pouvaient affronter le mal en 
manipulant Ryu Hayabusa (Ninja Gaiden, Tecmo) et ses "nouveaux pouvoirs et armements". Aux commandes de Shatterhand, "Aucune arme sur terre ne peut supporter le power dans vos mains ». Bien sûr, Nintendo convoquera aussi les super héros populaires. Dans l'adaptation du Batman de Tim Burton (Sunsoft, 1989), le chevalier noir est décrit comme «Incroyablement fort. Armé jusqu'aux dents [...] Et il n'arrêtera devant rien pour s'assurer que la justice l'emporte!». Les descriptions similaires abondent lorsqu'il s'agit d'amener les héros de bandes dessinées et de dessins animés dans un univers vidéoludique. Lors de sa première apparition sur Genesis, Spider-Man ne souffre plus de la même hésitation qu'à l'époque de la VCS : «Il donne des coups de poing et de pied avec une force spectaculaire ».

Bref, l'intégration de l' "équipement habilitant» au cœur des mécaniques de jeu a propulsé une accroche publicitaire axée sur la puissance à acquérir, d'abord destinée aux enfants de la génération Nintendo, et maintenant proférée à toutes les générations de joueurs.

\section{De l'aide en série}

La clarté et la limpidité qui émergent d'un système de règles abstrait constituent l'un des attraits fondamentaux de la pratique ludique. Un "bon design" permettrait au joueur de se plonger avec aisance dans un univers abstrait fortement régulé, mais qui reste suffisamment complexe pour assurer la découverte continuelle de nouvelles possibilités, et l'amélioration progressive des compétences du joueur. Comme l'ont souligné Juul, Salen et Zimmerman, le caractère procédural du jeu vidéo confère à ce dernier un avantage par rapport aux jeux traditionnels : le joueur peut apprendre les règles pendant que le système opère. Toutefois, le développement des jeux de progression complique significativement ce modèle; des règles distinctes - parfois uniques, comme les casse-têtes des jeux d'aventure - sont introduites progressivement tout au long de l'expérience, procurant au joueur un autre type de complexité à maitriser. Dans son livre What Video Games Have to Teach Us About Learning and Literacy (2003), James Paul Gee souligne la grande expertise développée par les créateurs de jeux pour faciliter l'apprentissage tout au long d'une expérience interactive, allant jusqu'à présenter le médium comme un exemple à suivre dans d'autres domaines. Nous présenterons maintenant l'émergence historique de diverses stratégies pour assister les joueurs lors de cette expérience d'apprentissage. Comme nous le verrons, la technologie a permis aux designers de perfectionner les stratégies d'assistance classique, et d'intégrer ces caractéristiques de manière plus ou moins visible au cœur même de l'expérience vidéoludique.

\section{Un apprentissage idéalisé}

Dans son livre, Gee étudie les stratégies qui visent à faciliter l'apprentissage dans des jeux contemporains comme System Shock 2 (Irrational Games, 1999) et Tomb Raider: The Last Revelation (Core Design, 1999). À l'époque où les systèmes de jeu étaient beaucoup plus simples, des éléments paratextuels fournissaient tout de même des directives claires pour faciliter la mise en action. Sur les machines d'arcade, les mécaniques essentielles ou innovantes étaient imprimées visiblement en périphérie de l'écran, avec l'intention évidente d'attirer les joueurs tout en leur enseignant celles-ci par la même 
occasion. Considérant la grande difficulté que nous avons soulignée plus haut, les instructions imprimées directement sur la machine Donkey Kong apparaîtront inévitablement ironiques: ces dernières précisent notamment que «Jump button makes Jumpman Jump» ("le bouton Saute permet à Jumpman de sauter»). La redondance d'informations entre les instructions écrites et celles affichées à l'écran durant le mode "attraction" était aussi une stratégie de tutorat commune. Dans Berzerk par exemple, les évènements montrés lors de la courte démonstration à l'écran font écho aux instructions écrites.

Contrastant avec le paradigme du «facile à apprendre, difficile à maîtriser », les premiers jeux de rôle édités sur ordinateur personnel étaient vendus avec la promesse d'univers étendus et variés à explorer. Basés sur les livres de règle de Dungeons and Dragons, ces jeux incluaient des manuels volumineux, remplis d'informations essentielles à la réussite de la quête. Les livrets détaillés sont devenus un aspect essentiel de l'expérience vidéoludique au cours des années 1980. Dans ce contexte, plusieurs stratégies d'assistance ont été perfectionnées. Tout au long de ses 30 pages, le manuel de Phantasie (SSI, 1985) est parsemé d' «indices de jeu ». Même le manuel d'un jeu d'action "simple" comme Batman: The Movie sur la NES intègre une description détaillée de chaque niveau, des sous-objectifs pour chacun, et des comportements typiques de tous les adversaires; le tout propose en définitive une sorte de « walkthrough » pour guider la performance du joueur.

Les didacticiels dynamiques, intégrés au cœur même de l'expérience interactive, représentent un développement majeur dans l'émergence du paradigme de design coopératif. En 1985, Gauntlet (Atari) a été conçu afin de populariser le dungeon crawler en dehors de sa niche établie sur microordinateurs. Afin de bien introduire certaines règles complexes au public de l'arcade, les designers ont créé un didacticiel élaboré qui s'étend sur plusieurs niveaux. Chaque fois qu'un nouvel élément entre en jeu, le système effectue une pause pour offrir des instructions textuelles, répercutées par une voix synthétique. Par exemple, cette interpellation directe invitait les joueurs à sauvegarder les potions " pour un usage ultérieur ", et l'arrivée des grunts au niveau 4 donne aussi lieu à des instructions spécifiques. Dans Thunder Blade (Sega, 1987), le premier niveau est clairement identifié comme une "mission d'entraînement »; 30 secondes sont offertes aux joueurs pour se familiariser avec les contrôles de l'hélicoptère. Gauntlet orchestre soigneusement l'ordre dans lequel les joueurs rencontrent les nouveaux objets ou ennemis; ce rythme d'apprentissage savamment orchestré définit pour l'essentiel ce que la communauté anglo-saxonne désigne sous l'appellation «tutorial levels ». À ce titre, les jeux antérieurs pouvaient également offrir de tels niveaux sans adresse manifeste ou étiquetage particulier. Même le terrifiant Sinistar laisse le joueur tranquille pendant une bonne minute avant de se déchaîner. Le fait d'amoindrir la courbe d'apprentissage au début de l'expérience envoie un signal coopératif, signal renforcé par une éventuelle mention explicite de la stratégie. Tout ceci fait écho au principe du «moratoire psychosocial » de Gee (2003, p. 222), où les apprenants "peuvent prendre des risques dans un espace où les conséquences associées au monde réel sont diminuées ».

En 2009, des millions de joueurs ont été invités à libérer Arkham Asylum (Rocksteady) de l'emprise du Joker. Durant une courte séquence introductive dans laquelle Batman escorte le célèbre vilain à l'intérieur de l'asile, le joueur apprend à naviguer et à regarder l'environnement, sans le moindre risque d'échec. Rapidement, le Joker 
s'échappe et libère quelques prisonniers pour ralentir Batman; il s'agit, bien sûr, de l'occasion idéale pour inciter le joueur à découvrir les premières mécaniques de combat. Lorsqu'ils feront face à leur premier ennemi extraordinaire (Bane), les joueurs n'auront pas eu le loisir de précipiter Batman vers la mort une seule fois avant de recevoir des indices textuels sur la façon de vaincre l'ennemi, et recevront des indices additionnels s'ils meurent. De nos jours, il est parfaitement habituel de voir des indices textuels prévus par les concepteurs surgir à l'écran lorsque les joueurs font face à certains nouveaux défis. L'assistance prend parfois la forme d'un rappel d'un élément du didacticiel: dans Crysis 2 (2011), par exemple, l'instruction " appuyez sur Q pour activer l'armure " apparaît pour éviter les chutes mortelles, et « appuyez sur C pour vous accroupir » pour signaler une issue moins évidente. Cette intégration dynamique d'indices correspond parfaitement au principe du «Juste au bon moment / information explicite sur demande » de Gee, où l'information est donnée « lorsque l'apprenant en a besoin, ou juste au moment où cette information sera le mieux comprise et utilisée en pratique » (Gee, 2003, p. 226).

\section{Un savoir en expansion}

Les jeux d'arcade classiques organisaient typiquement le monde de jeu en segments clairement divisés qui devaient être visités dans un ordre précis: vagues, niveaux, stages, etc. Dans Gran Trak 10, Space Invaders ou Crystal Castles, chaque segment est vu dans son entièreté à l'écran. Dans dnd (Gary Whisenhunt, Ray Wood, 1975) ou Colossal Cave Adventure (Will Crowther, 1977), un monde étendu et continu attend d'être exploré; les jeux de rôle et d'aventure reposeront en grande partie sur l'implémentation et la complexification de ce nouveau défi. Dans Temple of Apshai (Automated Simulations, 1979) et quelques autres pionniers du jeu de rôle, il devient essentiel de cartographier les donjons pour progresser de manière optimale. Le manuel de King's Quest (Sierra On-line, 1984) révèle qu'il " ne vous sera pas possible de compléter votre prophétie, Noble Graham, sans cartographier votre progrès ». Les développeurs de jeux ont commencé à offrir de l'assistance pour cette tâche en incluant dans le manuel quelques pages quadrillées (Alternate Reality: The City, Paradise Programming, 1986), ou même une large mappemonde à compléter avec des autocollants pré-identifiés au fur et à mesure que le joueur découvre de nouveaux lieux (The Legend of Blacksilver, Epyx, 1988). En 1985, Sword \& Sorcery sur le ZX Spectrum (Mike Simpson, Paul Hutchinson) intègre dans le même écran une vue d'ensemble du donjon ainsi qu'un point de vue à la première personne. En 1986, King's Quest 3: To Heir Is Human (Sierra) propose une « carte magique »; l'exploration du joueur y fait apparaître les nouveaux lieux. Westwood Associates ont implémenté l'auto-mapping (cartographie automatisée) depuis Mars Saga, jeu de rôle SF édité en 1988 ; l'écran y est divisé de manière à intégrer une carte abstraite qui fait état des déplacements du joueur en surplomb. Cette fonction sera rapidement disséminée dans l'ensemble des séries majeures - comme Might \& Magic (New World Computing) et The Bard's Tale (Electronic Arts).

30 L'expansion des univers de jeu a rapidement été suppléée par l'intégration de systèmes d'orientation spatio-narratifs à l'écran. Avec ces affichages dynamiques, les développeurs iront au-delà de la logique qui consiste à noter les éléments significatifs de l'espace ou des quêtes au fur et à mesure que le joueur rencontre ces éléments. Dans Battlezone, le joueur a déjà accès à un écran radar simple au-dessus de l'affichage 
principal, où des points rouges indiquent la position des ennemis proches. L'écran de surveillance de Defender (Williams, 1980) montre la totalité du niveau, ainsi que l'évolution dans l'espace des extraterrestres et des humains à protéger. Kapstone Kapers (Activision, 1983) utilise un système similaire, qui n'est plus cependant justifié par un univers de science-fiction ou la nature véhiculaire de l'avatar. À la même époque, Safecracker (Imagic, 1983) dirige les joueurs en faisant bon usage des feuillets transparents pour contrôleurs (propres à l'Atari 5200). Sur ces derniers, les directions cardinales sont associées à une couleur primaire. Alors qu'il évolue dans un monde ouvert présenté en vue isométrique, les bordures de l'écran changent de couleur afin de signifier la direction du prochain coffre à ouvrir. Dans The Legend of Zelda : A Link to the Past (Nintendo, 1991), les joueurs ont accès à une carte de vue d'ensemble, sur laquelle des icônes identifient la position actuelle de Link et la localisation de certains artefacts essentiels. Plusieurs jeux contemporains - des jeux de tir sur rails aux "carrés de sable " - font usage d'une carte dynamique superposée à l'écran de jeu qui garde constamment le joueur au fait des objectifs essentiels. Des jeux à monde ouvert comme The Simpsons : Hit \& Run (Radical, 2003) ou Red Faction: Guerilla (Volition, 2009), aux jeux plus linéaires et restreints comme Dead Space 2 (Visceral, 2011), des flèches ou des pistes apparaissent directement sur l'espace virtuel pour s'assurer que le joueur progresse de la manière la plus efficace possible.

En plus de ces éléments, il est essentiel de noter que le système d'orientation spationarratif comprend également plusieurs autres signes visuels qui apparaissent directement sur les représentations plus «naturelles» du monde virtuel. Dans un régime de restriction technologique, le design de jeu privilégiait les objets essentiels à la jouabilité, et ce faisant, proposait déjà des univers hautement fonctionnels et idéalisés. Le développement de moteurs visuels plus photoréalistes - qu'ils s'agissent de l'époque bitmap ou de la 3D polygonale - vient complexifier la lecture des jeux; la nécessité de hiérarchiser l'information justifie l'apparition de flèches directionnelles, de signalisations audiovisuelles abstraites, voire même d'un index superposé à l'image qui dirige le joueur au lieu de le punir (Double Dragon II : The Revenge, Technos, 1988). Le parallèle mérite d'être souligné à grands traits : à mesure que l'évolution technologique permet l'intégration d'univers audiovisuels luxuriants, le jeu vidéo voit apparaître toujours plus de signaux audiovisuels abstraits, ajoutant à la complexité apparente du déchiffrement, mais permettant en fait d'assurer une expérience plus fonctionnelle et gratifiante pour les joueurs.

\section{Baliser la progression}

En 1983, la boîte de Moon Patrol pour l'Atari 5200 souligne une "caractéristique unique » : la possibilité de continuer. Pour éviter la frustration de l'éternel retour en arrière, plusieurs jeux d'arcade des années 1980 offraient la possibilité de continuer au coût d'une pièce de monnaie, évidemment - depuis un point de sauvegarde tacite suivant la «mort» de l'avatar. Le monde de Wonder Boy (Sega, 1986) est découpé de manière similaire, chaque section étant identifiée par des nombres qui font office de checkpoints. Cette fonctionnalité sera largement implémentée durant l'ère de la NES, et les points de sauvegarde automatiques sont maintenant intégrés dans une majorité de jeux vidéo. Dans Super Mario World (Nintendo, 1991) ou Sonic the Hedgehog (Sega, 1991), les joueurs activent des marqueurs dans l'environnement; suivant la perte d'un Mario ou d'un Sonic, la prochaine «vie » se déploie à partir de ce point dans l'espace. Malgré 
une progression facilitée, ces jeux intègrent plusieurs des éléments de design que nous avons associés au paradigme de l'antagonisation, notamment un système de pointage et un nombre de vies limité. Lorsque ce système de «chances multiples" tombe à 0 , le niveau - voire le jeu en entier dans le cas de Sonic the Hedgehog - doit être recommencé du début. La présence de systèmes de sauvegardes automatisés ou tacites contribue grandement à faire du jeu vidéo une expérience "interruptible », aspect essentiel du jeu casual selon Juul (2009) : malgré une performance non optimale, l'investissement affectif et moteur du joueur "fructifie » et permet à ce dernier de progresser plus rapidement. Alors que la sauvegarde se pratique déjà plus librement sur microordinateur depuis le début des années 1980 (elle est nécessaire à l'économie ludique du jeu de rôle et du jeu d'aventure), l'émergence de supports de données réinscriptibles sur consoles permettra de démocratiser cette pratique.

Le point de sauvegarde se révélera salutaire pour permettre aux joueurs de progresser dans les univers vidéoludiques plus vastes. Dans le développement du paradigme coopératif que nous avons retracé tout au long de cet article, les concepteurs ont intégré plusieurs éléments qui permettent de signifier au joueur sa propre progression, parfois avec une insistance pointilliste. Plus spécifiquement, nous pensons à ce que l'on appelle communément en anglais les achievements et les collectibles, soit ces dizaines de défis additionnels qui viennent ponctuer la réalisation des objectifs cardinaux, ainsi que les nombreux objets à collectionner qui sont disséminés aux quatre coins de l'univers ludique. On peut observer l'intégration plus systématique de cette pratique depuis le début des années 1990 par l'utilisation de systèmes de pourcentage. Par exemple, Super Mario World affiche un nombre à la droite des fichiers de sauvegarde, dans le menu principal, comptabilisant le nombre de sorties trouvées par le joueur. Ce nombre actualise un méta-défi, en ce sens où le simple fait d'atteindre la fin du jeu ne suffit plus pour réellement compléter le jeu; pour ce faire, le joueur doit trouver tous les secrets. Finalement, il ne s'agit plus de «battre » le jeu, on cherche maintenant à «épuiser» les possibles, poussant encore plus loin la logique de la progression. Les niveaux comportant une sortie secrète sont d'ailleurs identifiés par un point rouge afin d'inciter une exploration plus systématique.

34 Afin de rendre compte du raffinement de cette stratégie de design, nous présenterons brièvement l'évolution des systèmes de collection et de complétion dans la série Donkey Kong Country sur SNES (Rare, 1994-1996). Comme Super Mario World, les trois opus proposent un pourcentage de complétion clairement affiché aux côtés des fichiers de sauvegarde. D'un jeu à l'autre, on observe une prolifération de secrets et d'objets cachés à trouver, ainsi qu'une aide grandissante afin d'effectuer le suivi de la progression. Dans le premier titre de la série, le manuel n'explique pas clairement les éléments liés au pourcentage. Ce dernier réfère au nombre de niveaux et de sous sections secrètes dispersés dans l'ensemble du jeu. Aucune indication ne vient préciser le nombre de "portes » - les barils-canons qui propulsent le joueur - qui mènent à ces sections. Déjà, le manuel du deuxième jeu (Donkey Kong Country 2: Diddy's Kong Quest) explique le fonctionnement du pourcentage, et un nouvel élément à collectionner est introduit: chaque niveau dissimule une médaille «DK». Le nombre de barils secrets sera comptabilisé par un affichage dynamique dans le troisième jeu(Donkey Kong Country 3: Dixie Kong's Double Trouble) : sur la mappemonde qui présente l'ensemble du parcours, le degré de complétion de chaque niveau est signifié par un drapeau; ce dernier se hisse lorsque le joueur découvre l'ensemble des secrets. Le suivi de la 
progression s'opère de manière constante, et perfectible pour un joueur dit " complétionniste ». Dans Donkey Kong 64 (Rare, 1999), la quantité d'objets à trouver et de micro-défis implémentés dans la quête du 100 \% est devenue si grande qu'elle a été qualifiée d'« écrasante " (Casamassina, 1999), ces tâches étant même perçues comme « du travail à accomplir » (Taruc, 1999). Finalement, on pourrait affirmer qu'à ce stade, ces objectifs en apparence secondaires semblent primer sur le simple fait d'atteindre la fin du jeu, devenant une figure représentative de ce que Salen et Zimmerman qualifient de conflit économique dans le jeu (2004, p. 14).

L'intégration de défis secondaires venant marquer la progression du joueur a pris en expansion ; le fabricant Microsoft l'impose dans les jeux édités sur sa console Xbox 360 à partir de 2005, et Sony fait écho à cette décision en 2008 avec le système des «trophées ». Entre-temps, Steam a également implémenté un système similaire - mais optionnel - sur sa plateforme de distribution numérique. Sur la plateforme de Microsoft, le Gamerscore est un système automatisé qui gère la totalité des réalisations d'une personne, information disponible sur le vaste réseau social Xbox Live. On retrouve ici plusieurs des traits essentiels du paradigme compétitif défini dans la première partie, mais la distinction est aussi frappante: le capital social n'est plus strictement accessible à une poignée de joueurs experts qui ont réussi à exploiter au mieux un système ludique particulièrement difficile à maitriser; ici, c'est toute la communauté des joueurs qui partage en ligne sa progression le long d'une liste de défis tantôt répétitifs (la collecte), tantôt incroyablement pointus (effectuer $\mathrm{x}$ actions à $\mathrm{x}$ moments au sein d'un contexte bien précis). Alors que le plaisir de la compétition sociale constitue l'un des attraits évidents de tels systèmes, ces derniers motivent les joueurs en accumulant des défis qui s'apparentent à ceux développés dans les jeux de progression dans une large mesure. Ces systèmes permettent par ailleurs aux joueurs de percevoir des traces extradiégétiques tangibles de leur progrès, qui se veut maintenant continuel.

\section{En guise de conclusion : un défi modulable}

Un dernier élément de design mérite d'être explicité afin de bien rendre compte du changement de paradigme documenté dans cet article: le défi modulable. Déjà, l'émergence des défis secondaires suppose une expérience plurielle, qui accommode différentes attitudes ludiques. Mais nous évoquons ici plus précisément la gestion extradiégétique de la performance par le joueur ou par le système. La sélection d'un niveau de difficulté clairement défini - typiquement : facile, moyen, difficile - émerge en fait à l'époque de l'arcade, et sera rapidement normalisée dans la production vidéoludique (Therrien, 2014). Cependant, cette sélection ne s'opère pas sans heurts dans Tempest (Atari, 1981) ou Star Wars (Atari, 1983) : le joueur qui choisit une vague ou un niveau plus facile pour commencer - équivalent du niveau «tutoriel » que nous avons évoqué plus haut - verra son score amputé d'un généreux bonus initial. Comme le souligne Ernst Adams (2008), la gestion tacite de la difficulté est apparue dès 1981 dans le jeu Astrosmash (Mattel Electronics). De nos jours, ce type d'altération automatisée de l'expérience en fonction de la performance du joueur est omniprésent, qu'il s'agisse de fournir plus d'indices en fonction de l'échec répété (Heart of Darkness, Amazing studio, 1998), de créer des pelletons artificiels dans un jeu de course (Mario 
Kart: Double Dash!!, Nintendo, 2003), ou de diminuer l'agressivité globale du système envers le joueur. Difficile toutefois de documenter avec précision ce type d'occurrence.

L'expérience vidéoludique semble globalement évoluer vers une plus grande flexibilité, pour reprendre l'un des critères essentiels du jeu casual proposé par Juul (2009). Or, cet élément de design émerge bien avant la "révolution» mise de l'avant par l'auteur. Nous avons pu constater que plusieurs des éléments de design que nous avons associés au paradigme d'assistance prennent place relativement tôt. La prémisse de Juul a déjà été nuancée par Lessard (2014) dans le cadre de son étude du jeu d'aventure graphique, et notre étude plus globale semble corroborer les conclusions de l'auteur. Ce dernier souligne les impératifs commerciaux qui favorisent l'émergence d'une plus grande accessibilité dans la seconde moitié des années 1980 : plusieurs acteurs de l'industrie constatent que ces jeux s'adressent à leurs semblables - soit des «hackers» ayant connu les premiers jeux d'aventure. La pérennité du genre reposera sur l'ouverture à un nouveau marché de joueurs non-initiés. C'est finalement cette contrainte commerciale qui semble dicter la plupart des évolutions ayant trait à l'accessibilité des jeux, comme notre parcours historique semble le confirmer.

Peu de temps après la révolution annoncée, faisant suite à des décennies d'évolution constante vers un paradigme de design coopératif, on observe un regain d'intérêt populaire pour une expérience punitive et intraitable. Une partie de la communauté de joueurs dénonce et ridiculise ouvertement les mesures d'assistance que nous avons présentées tout au long de cet article. La série qui émerge avec Demon's Souls (From Software, 2009) est emblématique de ce » retour aux sources ». Les mêmes voix qui évoquent un jeu " brutalement difficile » (VanOrd, 2009) ou encore " cruellement dur " (PS3M Staff, 2009) insistent du même souffle sur le caractère jouissif de l'expérience. Pour Sam Bishop (2009), les joueurs qui se souviennent du «bon vieux temps ", " alors que les jeux vous enseignaient à travers une savante utilisation de la punition intense et un prix élevé pour tout écart de conduite ", devraient se précipiter vers le jeu et laisser libre cours à ce masochisme vidéoludique.

Ultimement, nos recherches permettent d'observer un changement de paradigme, à partir d'une interpellation antagonisante jusqu'au design d'une expérience coopérative. Cependant, il serait faux de prétendre qu'une véritable dichotomie existe entre deux «moments» de l'histoire du jeu. On l'a bien constaté : des mécanismes d'assistance apparaissent déjà dans le contexte de l'arcade, et à ce jour un grand nombre de productions se targuent d'offrir un défi quasi-insurmontable en adoptant un discours publicitaire antagonisant. Ces deux approches ont plutôt évolué de manière parallèle et concomitante. L'hypothèse de la révolution casual mise de l'avant par Juul, qui prend acte de développements récents au niveau de la mise en marché des jeux, écarte plusieurs subtilités dans l'interaction entre les deux approches et leur évolution constante tout au long de l'histoire du jeu vidéo. Après le développement et la systématisation de plusieurs éléments de design qui tendent vers la coopération, les interactions récentes entre les circuits industriels et culturels semblent avoir rétabli l'équilibre en proposant des jeux parcourant un spectre plus large entre coopération et compétition. 


\section{BIBLIOGRAPHIE}

ADAMS E. (2008), « The Designer's Notebook: Difficulty Modes and Dynamic Difficulty Adjustment ", Gamasutra.com, 14 mai 2008.

ALEXANDER L. (2011), « GDC 2011 : McGonigal Says, “Don't Exploit Gamers” Extrinsic Rewards "Missing The Point" ", Gamasutra.com, 1er mars 2011.

BISHOP S. (2009), « Demon's Souls Review », IGN.com, 8 octobre 2009.

BURNHAM V. (2003), Supercade. A Visual History of the Videogame age 1971-1984, Cambridge, The MIT Press.

CASAMASSINA M. (1999), « Donkey Kong 64 », IGN.com, 24 novembre 1999.

CSIKSZENTMIHALYI M. (1975), Beyond Boredom and Anxiety. The Experience of Play in Work and Games, Washington, Jossey-Bass Publishers.

COLLECTIF (2009). « Demon’s Souls Review », Computerandvideogames.com, 30 nov. 2009.

DONOVAN T. (2010), Replay. The History of Video Games, East Sussex, Yellow Ant.

DUNCAN A. (2014), « Tex is back, older, but with a few new tricks up his sleeve », Destructoid.com, 9 mai 2014.

GEE J. P. (2003), What Video Games Have to Teach Us About Learning and Literacy, New York, Palgrave Macmillan.

HERMAN L. (1994), Phoenix: The Fall \& Rise of Videogames, Rolenta Press.

HUHTAMO E. (2012), « What's Victoria Got to Do With It », in Mark J. P. Wolf (dir.), Before the Crash : Early Video Game History, Detroit, Wayne State University Press, pp. 30-52.

JUUL J. (2005), Half-Real. Videogames between Real Rules and Fictional Worlds, Cambridge, the MIT Press.

JUUL J. (2009). A Casual Revolution : Reinventing Video Games and Their Players. Cambridge, The MIT Press.

JUUL J. (2013), The Art of Failure. Cambridge : The MIT Press.

KENT S. L. (2001), The Ultimate History of Video Games, New York, Three Rivers Press.

KOCUREK C. (2012), « Coin-Drop Capitalism : Economic Lessons from the Video Game Arcade », in Mark J. P. Wolf (dir.), Before the Crash : Early Video Game History, Detroit, Wayne State University Press, pp. 189-208.

KLINE S., DYER-WHITEFORD N. et DE PEUTER G. (2003), Digital Play: The Interaction of Technology, Culture, and Marketing, Montreal, McGill-Queen's University Press.

LESSARD J. (2014), « The Casual Revolution of 1987 », actes du 1er colloque international History of Games, Kinephanos.ca.

MONTFORT N. \& BOGOST I. (2009), Racing the Beam. The Atari Video Computer System, Cambridge, The MIT Press.

MORIARTY C. (2014), « Another World : 20th Anniversary Review », IGN.com, 11 juillet 2014.

REMO C. (2010), « GDC : Hecker's Nightmare Scenario - A Future Of Rewarding Players For Dull

Tasks », Gamasutra.com, 11 mars 2010. 
SALEN K. et ZIMMERMAN E. (2003), Rules of Play. Cambridge, The MIT Press.

TARUC N. (1999), « Donkey Kong 64 Review”, Gamespot.com, 22 nov. 1999.

THERRIEN C. (2012), « Video Games Caught Up In History. Accessibility, Teleological Distortion and Other Methodological Issues ", in Mark J. P. Wolf (dir.), Before the Crash : Early Video Game History, Detroit, Wayne State University Press, pp. 9-30.

THERRIEN C. (2014). « From the deceptively simple to the pleasurably complex. The rise of the cooperative address in video game design ", in H. Agius et M. Angelides (dir.) The Handbook of Digital Games, IEEE Press, pp. 548-74

VANORD K. (2009), « Demon's Souls Review », Gamestop.com, 6 oct. 2009.

WOLF M. J. P. (dir.) (2008), The Video Game Explosion. A History from PONG to PlayStation and Beyond. Westport, Greenwood Press.

WOLF M. J. P. (dir.) (2012), Encyclopedia of Video Games, Westport, Greenwood / ABC Clio Press, 2012.

\section{NOTES}

1. Toutes les citations en langue anglaise ont été traduites par les auteurs de ce texte.

2. De façon intéressante, la question des motivations intrinsèques et extrinsèques est devenue un sujet populaire dans les conventions industrielles des dernières années. Voir : Alexander (2011) et Remo (2010). Pour James Paul Gee (2003), les récompenses intrinsèques sont un aspect essentiel de sa typologie de l'apprentissage en 36 principes. Les structures des activités autoteliques - soit celles qui sont gratifiantes en elles-mêmes - ont été étudiées de façon notoire par Mihály Csíkszentmihályi (1975), et plusieurs des éléments qui seront discutés dans la seconde partie de ce texte sont directement reliés à des aspects soulignés par cet auteur.

\section{RÉSUMÉS}

Nous explorerons dans cet article l'évolution de l'antagonisation et de l'assistance dans le design des jeux vidéo à son ère industrielle. Cette exploration se fera en deux temps. Nous observerons d'abord l'émergence de la progression, à partir des jeux d'arcades classiques très itératifs aux jeux de plus grande ampleur, notant comment le défi et le discours antagonisant se modulent pour s'adapter à de nouvelles approches de design. Nous analyserons ensuite comment les mesures d'assistance apparaissent en parallèle afin de rendre l'expérience accessible à un plus large éventail de joueurs, favorisant ainsi l'atteinte de la fin du jeu. Au fil de ce parcours, nous verrons comment antagonisation et assistance sont en fait intimement liés en présentant quelques moments phares de cette évolution mutuelle.

In this article, we will explore the evolution of antagonization and assistance in video game design during its industrial period. To do so, we will divide our exploration in two sections. First, we will observe the rise of progression, from the iterative design of classic arcade games to games with a bigger scope, while noting how challenge and the antagonized discourses are 
evolving to cater to new design approaches. We will then analyze how assistance emerged in parallel in order to make the experience more accessible to a wider range of players, encouraging them to reach the end of the game. During the course of the article, we will see how antagonization and assistance are actually closely tied together by presenting a few key moments of this mutual evolution.

INDEX

Keywords : history of video games, design, difficulty, marketing, antagonization, cooperation Mots-clés : histoire du jeu vidéo, design, difficulté, marketing, antagonisation, coopération

\section{AUTEURS}

\section{CARL THERRIEN}

Université de Montréal

\section{MIKAËL JULIEN}

Université de Montréal 